\title{
NUMERICAL ANALYSIS OF PLANAR BICONE AND TEM HORN ARRAY ANTENNAS
}

\author{
Daniel T. McGrath and Carl E. Baum \\ USAF Phillips Laboratory \\ Kirtland AFB, NM
}

\section{INTRODUCTION}

Focused radiation of high power radio frequency (RF) transients has previously been achieved using reflector antennas fed by single spark-gap switches [1]. An attractive alternative would use an array of many medium-power sources, which are capable of lower pulse-to-pulse jitter than the spark gaps, and would offer the added advantage of time-delay scanning. This paper presents results of theoretical and numerical modeling of candidate radiating elements for ultrawideband (UWB) arrays. The elements examined are TEM horns that connect to one another at the mouths, and their limiting case, the planar bicone.

\section{PLANAR BICONE ARRAY}

Fig. 1 shows the geometry of a TEM horn array. The sources or loads are located at the apices, and the horns are joined to one another at their mouths. These connections are essential for low-frequency radiation because they permit a long "fill time." A TEM horn with an opening angle of $\beta=180^{\circ}$ is a planar bicone (PBC). It is an interesting starting point because, for a square lattice $\left(d_{x}=d_{y}\right)$, it is an approximation to a "self-complementary antenna," the exception being the non-point-source feed. Deschamps [2] showed that the input impedance of self-complementary antennas is $\eta_{d} / 2(60 \pi \mathrm{Ohms})$, and Baum [3] showed that the property also applies to self-complementary arrays at broadside scan. Hence, the planar bicone array is nearly optimum in terms of input impedance, although due to its bidirectionality it is not suitable for most practical applications.

In order to assess the effects of inter-element mutual coupling on the transmitting and receiving properties, an infinite $\mathrm{PBC}$ array was modeled using a frequency-domain hybrid finite element method that incorporates periodic boundary conditions [4]. This limits the analysis to a single unit cell. A receiving source was represented as a resistive load placed across the feed point. A typical unit cell model (a TEM horn with $\beta=120^{\circ}$ ) is shown in Fig. 2. The cells on the horn interior are removed to show the horn plates (shaded) and the resistive load (dark line). Periodicity conditions are imposed on the unit cell side walls in $x$ and $y$. Periodic radiation conditions are imposed on the front and back walls. The excitation is a plane wave incident from the $-z$ half space at an arbitrary angle $\left(\theta_{0} \phi_{0}\right)$.

The PBC's frequency response magnitude is shown in Fig. 3, with the frequency normalized to $f_{0}$ corresponding to $\lambda_{0}=d_{x}=d_{y}$. The curves for $30^{\circ}$ and $60^{\circ}$ scan are in the $\mathrm{H}$ plane--the E plane results are only slightly different [5].

At broadside scan, $50 \%$ of the incident power scatters nearly equally in forward and backscatter directions. At frequencies below $f_{0}$, the remaining $50 \%$ is absorbed by the array. However, as frequency increases through $f_{0}$, eight grating lobes enter visible space, four in each

\section{U.S. Government Work Not Protected by U.S. Copyright}


$\pm z$ half-space. The effect is that the array ceases to absorb any of the incident power, instead reflecting most of it in the grating lobe directions.

For off-broadside incidence, the results are similar above $f_{0}$. However, at the frequency $f_{G L}=f_{\delta} /\left(1+\sin \theta_{o}\right)$, a single pair of grating lobes enter visible space, one in each $\pm z$ half-space. This still causes a drop in the received power, although not as complete as the drop above $f_{0}$.

As a final note on the frequency response, this modeling approach, due to the infinite array assumption, does not accurately represent the low frequency behavior of an actual, finitesized array. Frequencies below that at which the array is less than one half wavelength across will not be absorbed on receive or radiated on transmit.

\section{PLANAR BICONE INPUT IMPEDANCE}

Using the same computer code to model the receiving response, the input impedance can be found by exploiting the fact that $Z_{i n}=V_{o c} I_{s c}$ and performing calculations with the load replaced alternately with open and short circuits. However, although the frequency response is fairly insensitive to the feed geometry, the input impedance is not. Hence, calculations for $Z_{\text {in }}$ were repeated using both original and refined feed geometries, shown in Figs. 4a and $4 \mathrm{~b}$. The refined feed more closely approximates a point source. The calculated results, shown in Fig. 5 , confirm that $Z_{i n}$ is very close to the self-complementary theory, being nearly independent of frequency, and within $20 \%$ of $\eta_{d} / 2$.

\section{TEM HORN ARRAY FREQUENCY RESPONSE}

The TEM horn is preferable to the planar bicone as an array element because it is directional, hence suitable for applications that have a structure on one side of the antenna. The calculated frequency response magnitude in Fig. 6 is, again, the received power due to a broadside-incident plane wave, measured across an $\eta d / 2$ load. The results are shown for three different flare angles and the PBC array, all with the same lattice dimensions. It is evident that in the low frequency limit, any TEM horn array, regardless of flare angle, behaves the same as a planar bicone array, with no directionality. The lower end of the frequency response improves as the horns are made longer. In addition, the response above $f_{0}$ improves due to the fact that (considering a transmit case) the horn's aperture field distribution is less able to couple into grating lobes.

\section{CONCLUSIONS}

This numerical exploration of the radiating properties of candidate broadband array elements has confirmed several important properties of the basic planar bicone and TEM hom elements. The PBC element, even with a practically-realizable feed, is a good approximation to a self-complementary array, having nearly constant $\eta_{d} / 2$ input impedance at broadside scan. TEM horn elements have identical characteristics in the low frequency limit, but at higher frequencies they sacrifice some uniformity in impedance for improved directionality. With both types of elements, the range of operating frequencies should be restricted to below that at which grating lobes can propagate. 


\section{REFERENCES}

1. Giri, David V. and Carl E. Baum, "Temporal and Spectral Radiation on Boresight of a Reflector Type of Impulse Radiating Antenna (IRA)." Ultra-Wideband, Short-Pulse Electromagnetics 3, ed.

C.E. Baum, L. Carin and A.P. Stone, Plenum Press, 1997.

2. Deschamps, Georges A., "Impedance Properties of Complementary Multiterminal Planar Structures," IRE Trans. Antennas Propagat., pp. S371-S378, Dec. 1959.

3. Baum, Carl E., "Early Time Performance at Large Distances of Periodic Planar Arrays of Planar Bicones with Sources Triggered in a Plane-Wave Sequence," Sensor and Simulation Note 184, Aug.

1973.

4. McGrath, Daniel T. and Vittal P. Pyati, "Periodic structure analysis using a hybrid finite element method," Radio Science, 31, pp. 1173-1179, Sep.-Oct. 1996.

5. McGrath, Daniel T., "Numerical Analysis of Planar Bicone Arrays," Sensor and Simulation Note \#403, Dec. 1996.

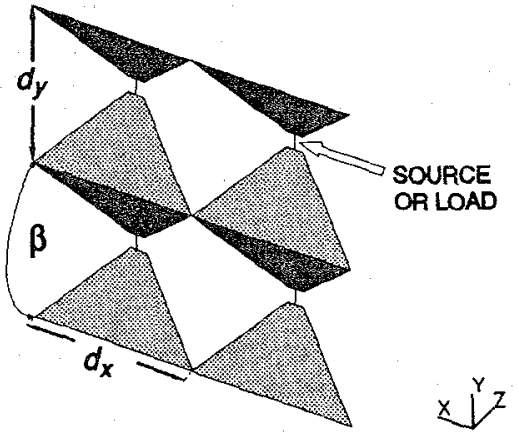

Figure 1. TEM Horn Array Geometry

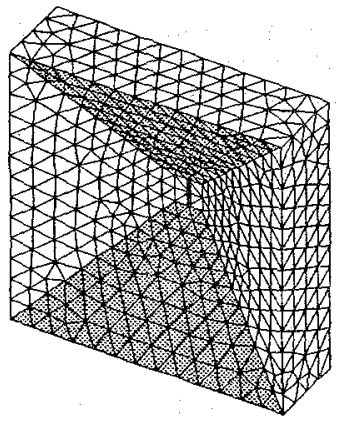

Figure 2. Typical Finite Element Model

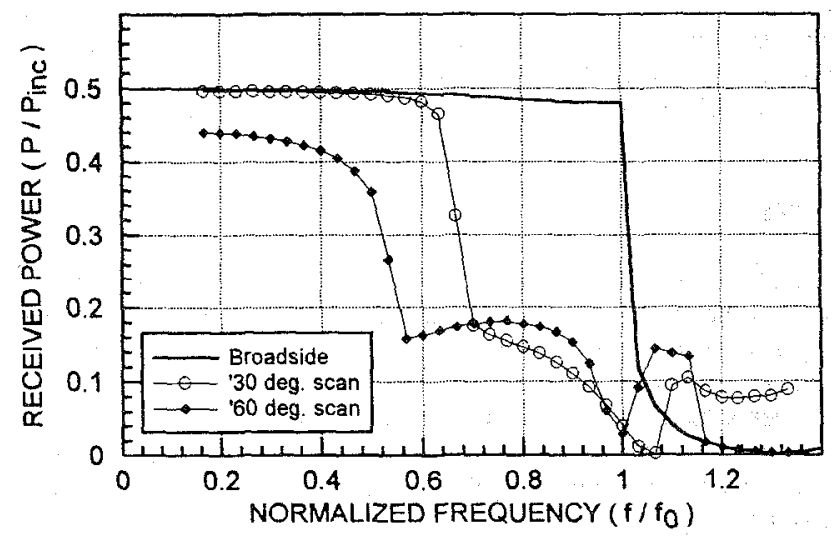

Figure 3. Planar Bicone Array Frequency Response Magnitude $(0,30$ and 60 degrees incidence in $\mathrm{H}$ plane $)$ 


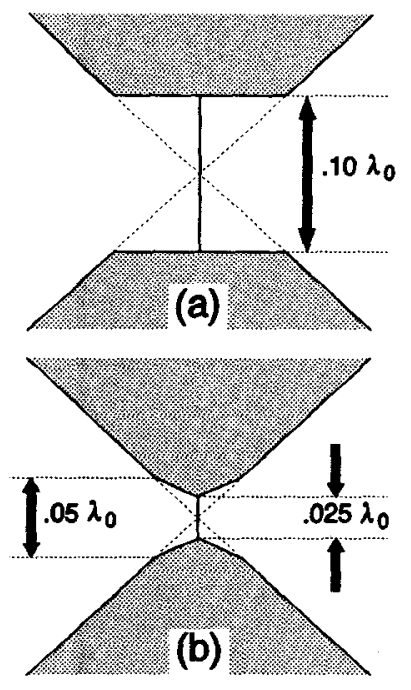

Figure 4. Planar Bicone Array Feed Geometries

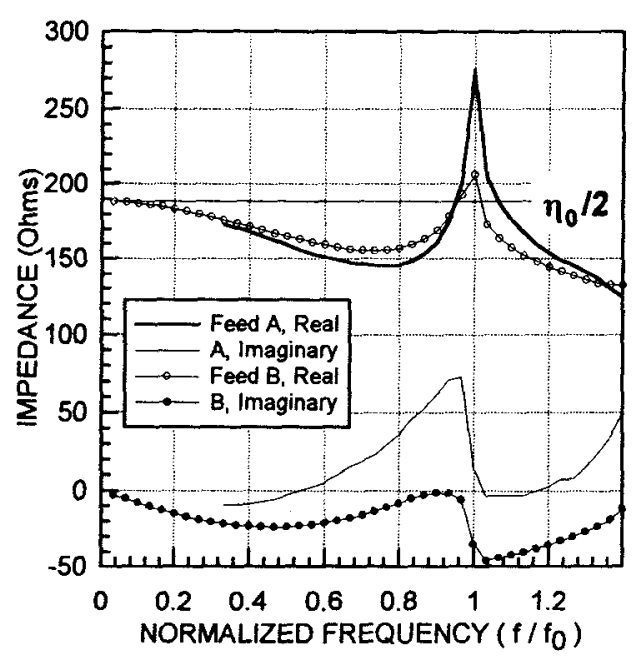

Figure 5. Planar Bicone Array Input Impedance with Feed Models from Fig. 4

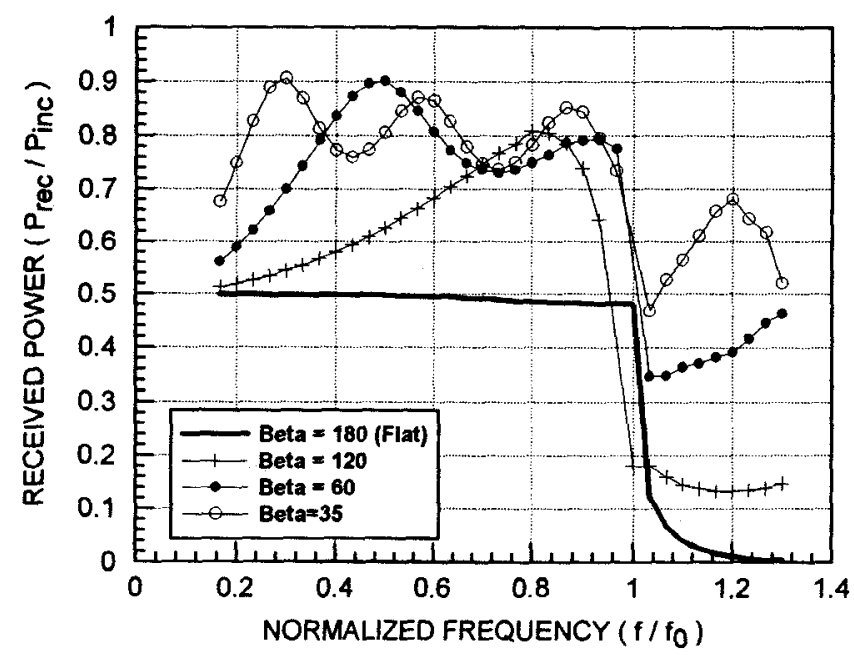

Figure 6. TEM Horn Array Frequency Response Magnitude 